\title{
Nanometer-Scale Structures of Neurons Differ Between Individuals and Those Differences Become Extraordinary in Schizophrenia
}

Rino Saiga ${ }^{1}$, Vincent De Andrade ${ }^{2}$, Francesco De Carlo ${ }^{2}$, Akihisa Takeuchi ${ }^{3}$, Kentaro Uesugi $^{3}$, Yasuko Terada $^{3}$, Yoshio Suzuki ${ }^{4}$, Susumu Takekoshi ${ }^{5}$, Chie Inomoto ${ }^{5}$, Naoya Nakamura ${ }^{5}$, Youta Torii ${ }^{6}$, Itaru Kushima $^{6}$, Shuji Iritani ${ }^{6}$, Norio Ozaki ${ }^{6}$, Kenichi Oshima ${ }^{7}$, Masanari Itokawa ${ }^{7}$, Makoto Arai ${ }^{7}$ and Ryuta Mizutani $^{1 *}$

1. Department of Applied Biochemistry, Tokai University, Hiratsuka, Kanagawa, Japan.

2. Advanced Photon Source, Argonne National Laboratory, Lemont, IL, USA.

3. Japan Synchrotron Radiation Research Institute (JASRI/SPring-8), Sayo, Hyogo, Japan.

4. Graduate School of Frontier Sciences, University of Tokyo, Kashiwa, Chiba, Japan.

5. Tokai University School of Medicine, Isehara, Kanagawa, Japan.

6. Graduate School of Medicine, Nagoya University, Nagoya, Aichi, Japan.

7. Tokyo Metropolitan Institute of Medical Science, Setagaya, Tokyo, Japan.

* Corresponding author: mizutanilaboratory@gmail.com

The brain consists of a huge number of neurons forming micrometer- to nanometer-scale networks. This study reports a three-dimensional analysis of brain neurons of schizophrenia sufferers and control cases using synchrotron radiation nanotomography. The results indicate that neuronal structures differ between individuals, and those differences become extraordinary in schizophrenia. We suggest that such structural differences in neurons reflect the mental individuality of humans [1].

The cerebral tissues used in this study were taken from the anterior cingulate cortex. It has been reported that this brain area is responsible for higher brain functions, including decision-making and is related to psychiatric diseases, such as schizophrenia and autism. The brain tissues were impregnated with Golgi staining in order to visualize the neurons in x-ray images [2]. The stained tissues were embedded in epoxy resin and subjected to nanotomography. The study was conducted according to the procedure approved by the ethics committees of the related organizations.

Synchrotron radiation nanotomography was performed at the 32-ID beamline of the Advanced Photon Source of Argonne National Laboratory and at the BL37XU and the BL47XU beamlines of SPring-8. The resin embedded samples were mounted on precision rotation stages specially built for nanotomography. Transmission images of the brain tissues were taken with Fresnel zone plate optics using monochromatic radiation of $8 \mathrm{keV}$. The images were processed with the convolution backprojection method to reconstruct the three-dimensional structures of the neurons. The spatial resolutions of the reconstructed images were estimated to be 180-300 nm using square-wave patterns [3] and the Fourier domain plot $[4,5]$.

In order to analyze the structural differences of the neurons, we traced three-dimensional images of neurons to build Cartesian coordinate models of the neuronal structures. The initial models were generated from the images by using machine recognition algorithms. The computer generated models were refined with conjugate gradient minimization. Examples of neuronal structures of schizophrenia and control cases are shown in Figure 1. Since these models are represented with Cartesian coordinates, the structural differences of the neurons can be examined quantitatively by calculating their geometrical parameters. In particular, neurites can be regarded as three-dimensional curves, which are represented 
with two geometrical parameters: curvature and torsion. Curvature is the reciprocal of the radius of the curve. Torsion is the deviation of the three-dimensional curve from a plane. The analysis of these geometrical parameters of the neurites revealed that the median of neurite curvature was significantly different between individuals, even within the control cases $\left(p<2.2 \times 10^{-16}\right.$ with a Kruskal-Wallis test). Multiple samples from the same individual showed similar frequency distributions of curvature, suggesting that the neurite curvature is inherent to each individual. We also analyzed structural differences between the schizophrenia and control cases. The neurites of the schizophrenia cases showed extraordinarily high curvatures, resulting in tortuous structures (Figure 1a), while the neurites of the control cases were rather straight (Figure 1b). The mean curvature of the schizophrenia cases was 1.5times higher than that of the control cases $(p=0.020$, two-sided Welch's t-test for 4 schizophrenia and 4 control cases). Since the neurite geometry determines the spatial trajectory of the neurites, these results indicate that the neuronal microcircuits of the schizophrenia cases were different from those of the controls.

The brain area analyzed in this study was the anterior cingulate cortex that is responsible for higher brain functions. Structural differences found in the neurons of this area can affect neuronal microcircuits involved in mental activity. We suggest that the nanometer-scale differences in neuronal structure revealed in this study reflect the individuality of our minds and thoughts $[1,6]$.

\section{References:}

[1] R Mizutani et al., Transl. Psychiatry 9 (2019), p. 85. https://doi.org/10.1038/s41398-019-0427-4.

[2] R Mizutani et al., Brain Res. 1199 (2008), p. 53.

[3] R Mizutani et al., J. Synchrotron Radiation 15 (2008), p. 648.

[4] R Mizutani et al., J. Microscopy 261 (2016), p. 57.

[5] R Saiga et al., Micron 105 (2018), p. 64.

[6] The synchrotron radiation experiments at the Advanced Photon Source were performed under GUP45781 and GUP-59766. This research used resources of the Advanced Photon Source, a U.S.

Department of Energy (DOE) Office of Science User Facility operated for the DOE Office of Science by Argonne National Laboratory under Contract No. DE-AC02-06CH11357. The synchrotron radiation experiments at the SPring-8 were performed with the approval of JASRI (2013B0041, 2014A1057, 2015B1101, 2017A1143, and 2018B1187). This work was supported by Grants-in-Aid for Scientific Research from the JSPS (21611009, 25282250, and 25610126).

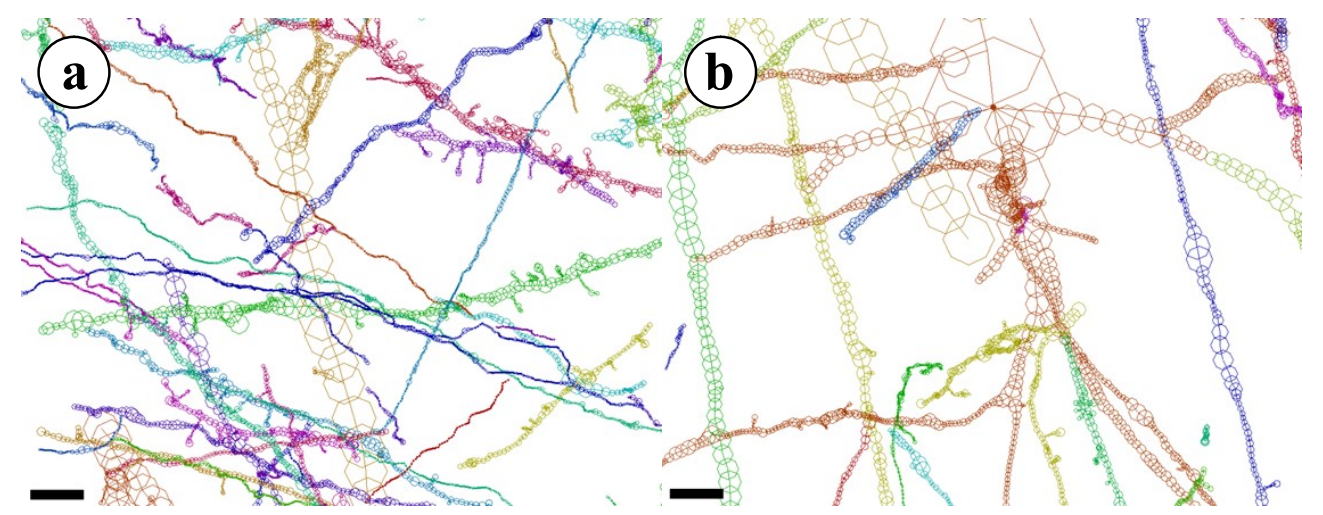

Figure 1. Neuronal network of (a) schizophrenia and (b) control cases. Structures are color-coded. Brain surface is toward the top. Scale bars: $5 \mu \mathrm{m}$. 